\title{
Erratum to: Oscillation of second-order delay dynamic equations on time scales
}

\author{
Shurong Sun • Zhenlai Han • Tongxing Li
}

Received: 4 September 2009 / Published online: 18 April 2012

(C) Korean Society for Computational and Applied Mathematics 2012

\section{Erratum to: J Appl Math Comput (2009) 30: 459-468 DOI 10.1007/s12190-008-0185-6}

In the above-referenced paper [1], we stated and proved four theorems on the sufficient conditions for the oscillation of all solutions of second order delay dynamic equations

$$
\left(p(t)\left(x^{\Delta}(t)\right)^{\gamma}\right)^{\Delta}+q(t) f(x(\tau(t)))=0
$$

on a time scale $\mathbb{T}$, where $\gamma \geq 1$ is a quotient of odd positive integers, $p$ and $q$ are positive, real-valued rd-continuous functions defined on $\mathbb{T}, \tau: \mathbb{T} \rightarrow \mathbb{T}$ is a strictly

The online version of the original article can be found under doi:10.1007/s12190-008-0185-6.

This research is supported by the Natural Science Foundation of China (11071143, 60904024, 61174217), Natural Science Outstanding Youth Foundation of Shandong Province (JQ201119) and supported by Shandong Provincial Natural Science Foundation (ZR2010AL002, ZR2009AL003), also supported by Natural Science Foundation of Educational Department of Shandong Province (J11LA01).

S. Sun $\cdot$ Z. Han $(\bowtie) \cdot$ T. Li

School of Mathematical Sciences, University of Jinan, Jinan, Shandong 250022, P.R. China

e-mail: hanzhenlai@163.com

T. Li

e-mail: litongx2007@163.com

S. Sun

Department of Mathematics and Statistics, Missouri University of Science and Technology, Rolla, MO 65409-0020, USA

e-mail: sshrong@163.com

Z. Han

School of Control Science and Engineering, Shandong University, Jinan, Shandong 250061,

P.R. China 
increasing function such that $\tau(t) \leq t$ and $\tau(t) \rightarrow \infty$ as $t \rightarrow \infty, f \in C(\mathbb{R}, \mathbb{R})$ which satisfies for some positive constant $L, \frac{f(x)}{x^{\gamma}} \geq L$, for all nonzero $x$. There were mistakes in the statements of the four theorems, and we wish to correct the mistakes below.

We define the time scale interval of the form $\left[t_{0}, \infty\right)_{\mathbb{T}}$ by $\left[t_{0}, \infty\right)_{\mathbb{T}}=\left[t_{0}, \infty\right) \cap \mathbb{T}$, and consider the two cases

$$
\int_{t_{0}}^{\infty}\left(\frac{1}{p(t)}\right)^{\frac{1}{\gamma}} \Delta t=\infty
$$

and

$$
\int_{t_{0}}^{\infty}\left(\frac{1}{p(t)}\right)^{\frac{1}{\gamma}} \Delta t<\infty
$$

The four theorems are corrected as follows:

Theorem 1 Assume $(0.2)$ holds and $\tau \in C_{r d}^{1}\left(\left[t_{0}, \infty\right)_{\mathbb{T}}, \mathbb{T}\right), \widetilde{\mathbb{T}}:=\tau(\mathbb{T}) \subset \mathbb{T}$ is a time scale, $\tau(\sigma(t))=\sigma(\tau(t))$. Furthermore, assume that there exists a positive function $\delta \in C_{r d}^{1}\left(\left[t_{0}, \infty\right)_{\mathbb{T}}, \mathbb{R}\right)$ such that

$$
\limsup _{t \rightarrow \infty} \int_{t_{0}}^{t}\left(L q(s) \delta(s)-\frac{p(\tau(s))\left(\left(\delta^{\Delta}(s)\right)_{+}\right)^{\gamma+1}}{(\gamma+1)^{\gamma+1}\left(\delta(s) \tau^{\Delta}(s)\right)^{\gamma}}\right) \Delta s=\infty,
$$

where $\left(\delta^{\Delta}(s)\right)_{+}=\max \left\{0, \delta^{\Delta}(s)\right\}$. Then $(0.1)$ is oscillatory on $\left[t_{0}, \infty\right)_{\mathbb{T}}$.

Theorem 2 Assume (0.2) holds and $\tau \in C_{r d}^{1}\left(\left[t_{0}, \infty\right)_{\mathbb{T}}, \mathbb{T}\right), \widetilde{\mathbb{T}}:=\tau(\mathbb{T}) \subset \mathbb{T}$ is a time scale, $\tau(\sigma(t))=\sigma(\tau(t))$. Furthermore, assume that there exists a positive function $\delta \in C_{r d}^{1}\left(\left[t_{0}, \infty\right)_{\mathbb{T}}, \mathbb{R}\right)$ such that for $m \geq 1$

$$
\limsup _{t \rightarrow \infty} \frac{1}{t^{m}} \int_{t_{0}}^{t}(t-s)^{m}\left(L q(s) \delta(s)-\frac{p(\tau(s))\left(\left(\delta^{\Delta}(s)\right)_{+}\right)^{\gamma+1}}{(\gamma+1)^{\gamma+1}\left(\delta(s) \tau^{\Delta}(s)\right)^{\gamma}}\right) \Delta s=\infty,
$$

where $\left(\delta^{\Delta}(s)\right)_{+}=\max \left\{0, \delta^{\Delta}(s)\right\}$. Then $(0.1)$ is oscillatory on $\left[t_{0}, \infty\right) \mathbb{T}$.

Theorem 3 Assume $(0.3)$ holds and $\tau \in C_{r d}^{1}\left(\left[t_{0}, \infty\right)_{\mathbb{T}}, \mathbb{T}\right), \widetilde{\mathbb{T}}:=\tau(\mathbb{T}) \subset \mathbb{T}$ is a time scale, $\tau(\sigma(t))=\sigma(\tau(t))$. Furthermore, assume that there exists a positive function $\delta \in C_{r d}^{1}\left(\left[t_{0}, \infty\right)_{\mathbb{T}}, \mathbb{R}\right)$ such that $(0.4)$ holds. If

$$
\int_{t_{0}}^{\infty}\left[\frac{1}{p(t)} \int_{t_{0}}^{t} q(s) \Delta s\right]^{\frac{1}{\gamma}} \Delta t=\infty
$$

then every solution of $(0.1)$ is either oscillatory or converges to zero on $\left[t_{0}, \infty\right)_{\mathbb{T}}$.

Theorem 4 Assume $(0.3)$ holds and $\tau \in C_{r d}^{1}\left(\left[t_{0}, \infty\right) \mathbb{T}, \mathbb{T}\right), \widetilde{\mathbb{T}}:=\tau(\mathbb{T}) \subset \mathbb{T}$ is a time scale, $\tau(\sigma(t))=\sigma(\tau(t))$. Furthermore, assume that there exists a positive function $\delta \in C_{r d}^{1}\left(\left[t_{0}, \infty\right)_{\mathbb{T}}, \mathbb{R}\right)$ such that $(0.5)$ and $(0.6)$ hold. Then every solution of $(0.1)$ is either oscillatory or converges to zero on $\left[t_{0}, \infty\right) \mathbb{T}$. 
Actually, the major results are based on Lemma 2.2 (p. 462, line 26). We think these are not useful in this case because the forward jump operator $\widetilde{\sigma}$ in $\widetilde{\mathbb{T}}$ is not the same $\sigma$ in $\mathbb{T}$, so the differentiation in p. 463 line 19 is not correct and also $(x(\tau(t)))^{\Delta}=x^{\Delta}(\tau(t)) \tau^{\Delta}(t)$ is not correct in general. Therefore, we are intend to correct the mistakes as follows.

Lemma (Chain Rule) Assume that $\tau: \mathbb{T} \rightarrow \mathbb{R}$ is strictly increasing and $\widetilde{\mathbb{T}}:=\tau(\mathbb{T}) \subset$ $\mathbb{T}$ is a time scale, $\tau(\sigma(t))=\sigma(\tau(t))$. Let $x: \widetilde{\mathbb{T}} \rightarrow \mathbb{R}$. If $\tau^{\Delta}(t)$ and $x^{\Delta}(\tau(t))$ exist for $t \in \mathbb{T}^{\kappa}$, then $(x(\tau(t)))^{\Delta}$ exists, and

$$
(x(\tau(t)))^{\Delta}=x^{\Delta}(\tau(t)) \tau^{\Delta}(t) .
$$

Proof Let $0<\varepsilon<1$ be given and define $\varepsilon^{*}=\varepsilon\left[1+\left|\tau^{\Delta}(t)\right|+\left|x^{\Delta}(\tau(t))\right|\right]^{-1}$. Note that $0<\varepsilon^{*}<1$. According to the assumptions, there exist neighborhoods $\mathcal{N}_{1}$ of $t$ and $\mathcal{N}_{2}$ of $\tau(t)$ such that

$$
\left|\tau(\sigma(t))-\tau(s)-(\sigma(t)-s) \tau^{\Delta}(t)\right| \leq \varepsilon^{*}|\sigma(t)-s|, \quad s \in \mathcal{N}_{1}
$$

and

$$
\left|x(\sigma(\tau(t)))-x(r)-(\sigma(\tau(t))-r) x^{\Delta}(\tau(t))\right| \leq \varepsilon^{*}|\sigma(\tau(t))-r|, \quad r \in \mathcal{N}_{2} .
$$

Put $\mathcal{N}=\mathcal{N}_{1} \cap \tau^{-1} \mathcal{N}_{2}$ and let $s \in \mathcal{N}$. Then $s \in \mathcal{N}_{1}$ and $\tau(s) \in \mathcal{N}_{2}$ and

$$
\begin{aligned}
\mid x( & (\sigma(t)))-x(\tau(s))-(\sigma(t)-s) x^{\Delta}(\tau(t)) \tau^{\Delta}(t) \mid \\
= & \mid x(\tau(\sigma(t)))-x(\tau(s))-(\sigma(\tau(t))-\tau(s)) x^{\Delta}(\tau(t)) \\
& +\left[\sigma(\tau(t))-\tau(s)-(\sigma(t)-s) \tau^{\Delta}(t)\right] x^{\Delta}(\tau(t)) \mid \\
= & \mid x(\sigma(\tau(t)))-x(\tau(s))-(\sigma(\tau(t))-\tau(s)) x^{\Delta}(\tau(t)) \\
& +\left[\tau(\sigma(t))-\tau(s)-(\sigma(t)-s) \tau^{\Delta}(t)\right] x^{\Delta}(\tau(t)) \mid \\
\leq & \varepsilon^{*}|\sigma(\tau(t))-\tau(s)|+\varepsilon^{*}|\sigma(t)-s|\left|x^{\Delta}(\tau(t))\right| \\
\leq & \varepsilon^{*}\left\{\left|\sigma(\tau(t))-\tau(s)-(\sigma(t)-s) \tau^{\Delta}(t)\right|\right. \\
& \left.+|\sigma(t)-s|\left|\tau^{\Delta}(t)\right|+|\sigma(t)-s|\left|x^{\Delta}(\tau(t))\right|\right\} \\
= & \varepsilon^{*}\left\{\left|\tau(\sigma(t))-\tau(s)-(\sigma(t)-s) \tau^{\Delta}(t)\right|\right. \\
& \left.+|\sigma(t)-s|\left|\tau^{\Delta}(t)\right|+|\sigma(t)-s|\left|x^{\Delta}(\tau(t))\right|\right\} \\
\leq & \varepsilon^{*}\left\{\varepsilon^{*}|\sigma(t)-s|+|\sigma(t)-s|\left|\tau^{\Delta}(t)\right|+|\sigma(t)-s|\left|x^{\Delta}(\tau(t))\right|\right\} \\
= & \varepsilon^{*}|\sigma(t)-s|\left\{\varepsilon^{*}+\left|\tau^{\Delta}(t)\right|+\left|x^{\Delta}(\tau(t))\right|\right\} \\
\leq & \varepsilon^{*}|\sigma(t)-s|\left\{1+\left|\tau^{\Delta}(t)\right|+\left|x^{\Delta}(\tau(t))\right|\right\} \\
= & \varepsilon|\sigma(t)-s| .
\end{aligned}
$$

The proof is complete. 


\section{References}

1. Sun, S., Han, Z., Zhang, C.: Oscillation of second-order delay dynamic equations on time scales. J. Appl. Math. Comput. 30, 459-468 (2009) 